Supporting Information

\title{
Dominant Factors Governing the Electron Transfer Kinetics and Electrochemical Biosensing Properties of Carbon Nanofiber Arrays
}

Liangsheng Hu, ${ }^{\dagger, \S, 1}$ Xiang Peng, ${ }^{\Perp l, 1}$ Kaifu Huo, ${ }^{* \dagger}$ Rongsheng Chen, ${ }^{\ddagger}$ Jijiang Fu,

Yong Li, ${ }^{\S}$ Lawrence Yoon Suk Lee, ${ }^{\S}$ Kwok-Yin Wong ${ }^{*}$, and Paul K Chu ${ }^{*}, \|$

${ }^{\dagger}$ Wuhan National Laboratory for Optoelectronics (WNLO) and School of Optical and Electronic Information, Huazhong University of Science and Technology, Wuhan 430074, China. E-mail: kfhuo@hust.edu.cn

${ }^{\ddagger}$ The State Key Laboratory of Refractories and Metallurgy, Wuhan University of Science and Technology, Wuhan 430081, China

"Department of Physics and Materials Science, City University of Hong Kong, Tat Chee Avenue, Kowloon, Hong Kong, China. E-mail: paul.chu@ cityu.edu.hk

${ }^{\S}$ Department of Applied Biology and Chemical Technology and the State Key Laboratory of Chirosciences, The Hong Kong Polytechnic University, Hung Hom, Hong Kong, China. E-mail: kwok-yin.wong@polyu.edu.hk

${ }^{1}$ These authors contributed equally to this work 

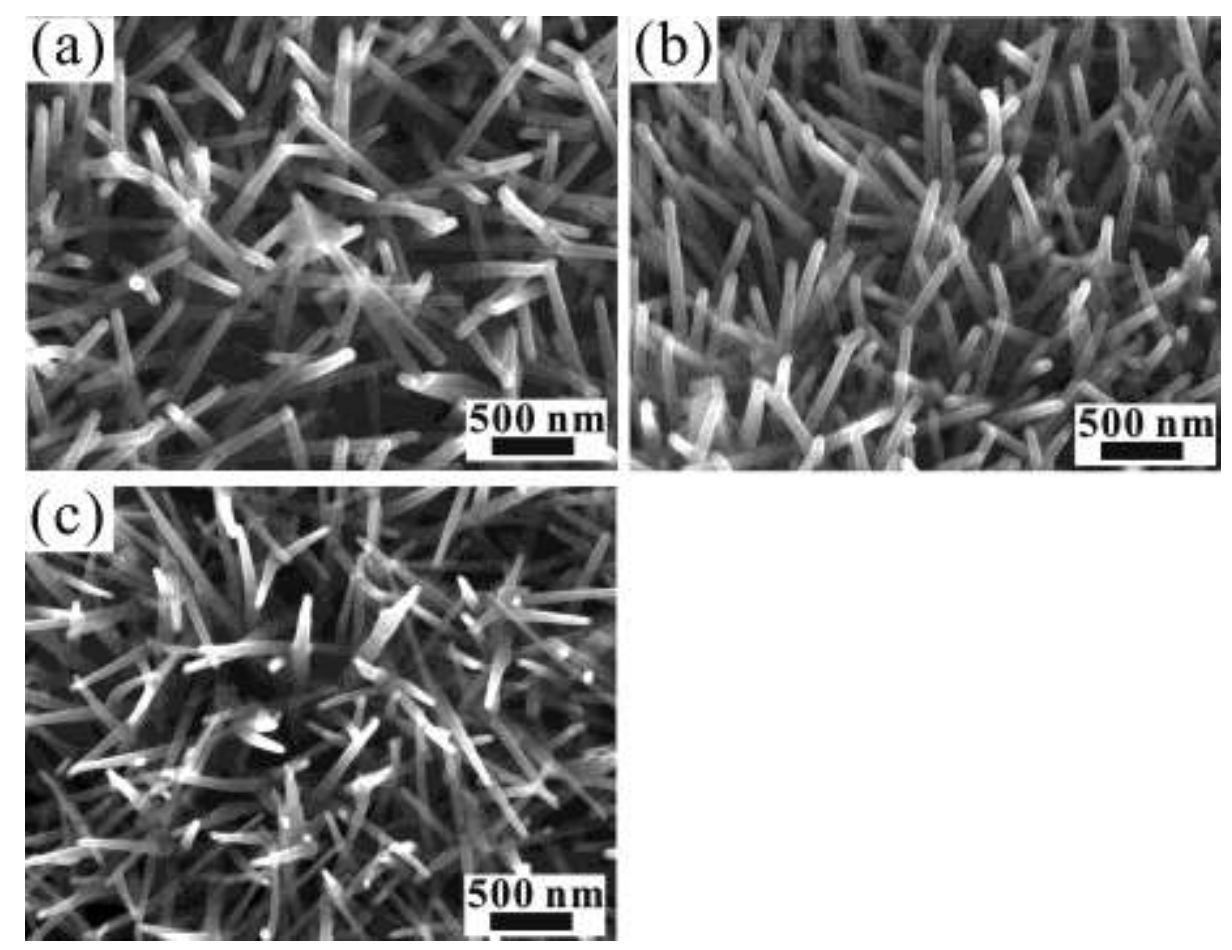

Figure S1. Representative enlarged FE-SEM images of the cylindrical (a) $\mathrm{TiO}_{2} / \mathrm{C}$ and (b) $\mathrm{TiC} / \mathrm{C}$, and (c) conical TiC/C QANFAs.

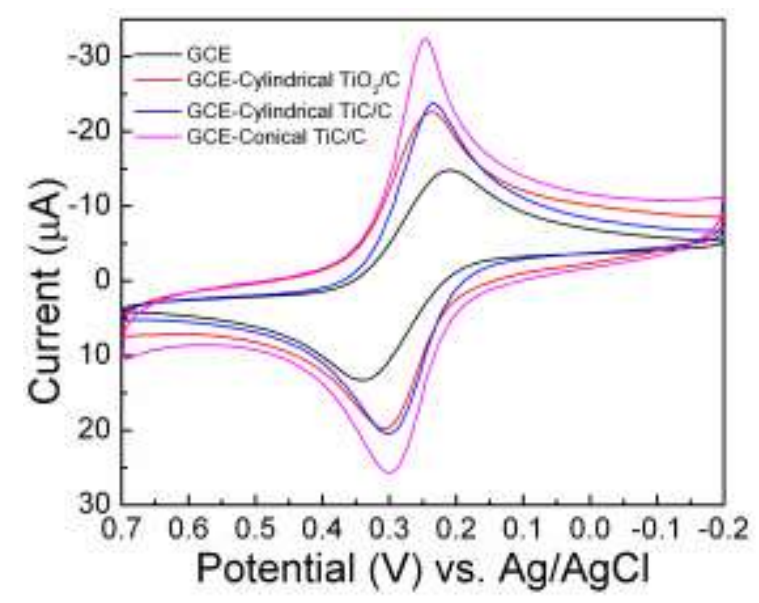

Figure S2. Cyclic voltammograms $(\mathrm{CVs})$ of $1.0 \mathrm{mM} \mathrm{K}_{3} \mathrm{Fe}(\mathrm{CN})_{6}$ in a $1.0 \mathrm{M} \mathrm{KCl}$ solution at a scanning rate of $100 \mathrm{mV} \mathrm{s}^{-1}$ from the glassy carbon electrode (GCE, black), cylindrical $\mathrm{TiO}_{2} / \mathrm{C}$ (red), $\mathrm{TiC} / \mathrm{C}$ (blue), and conical $\mathrm{TiC} / \mathrm{C}$ (purple) nanofiber-modified GCEs. 

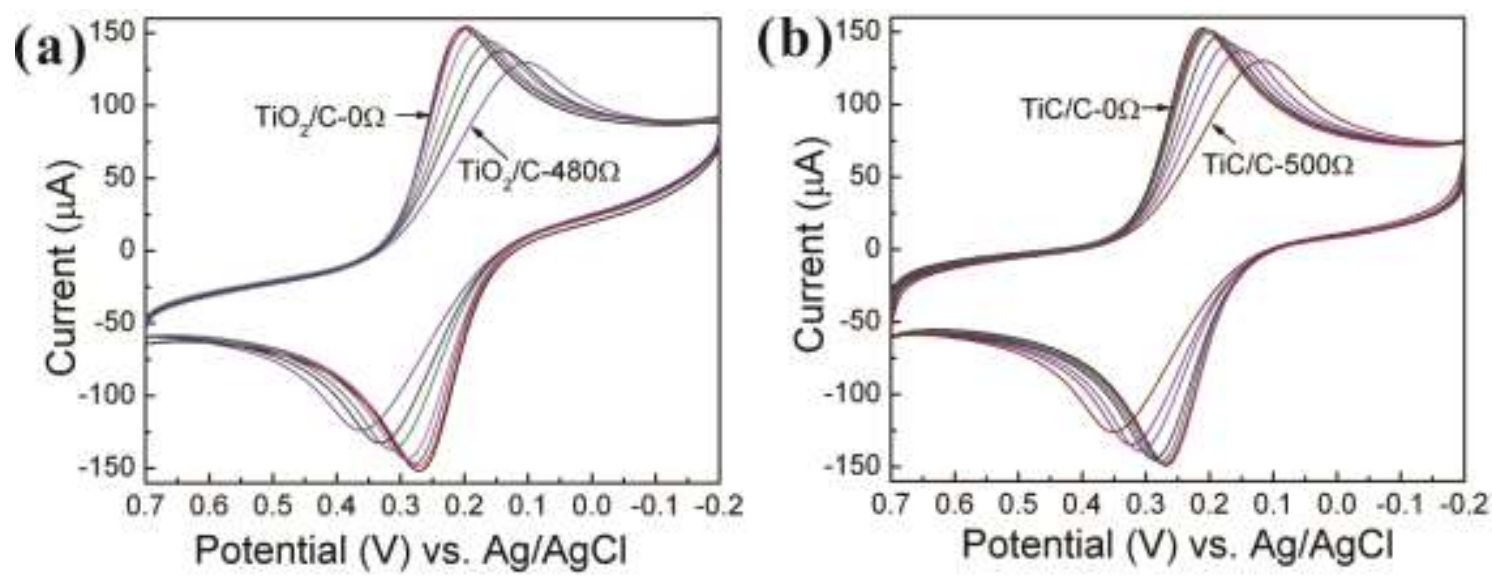

Figure S3. CVs obtained from $1.0 \mathrm{mM} \mathrm{K}_{3} \mathrm{Fe}(\mathrm{CN})_{6}$ in $1.0 \mathrm{M} \mathrm{KCl}$ at a scanning rate of $100 \mathrm{mV} \mathrm{s}^{-1}$ for (a) cylindrical $\mathrm{TiC} / \mathrm{C}$ connected to $0-480 \Omega$ resistors and (b) $\mathrm{TiC} / \mathrm{C}$ connected to $0-500 \Omega$ resistors.

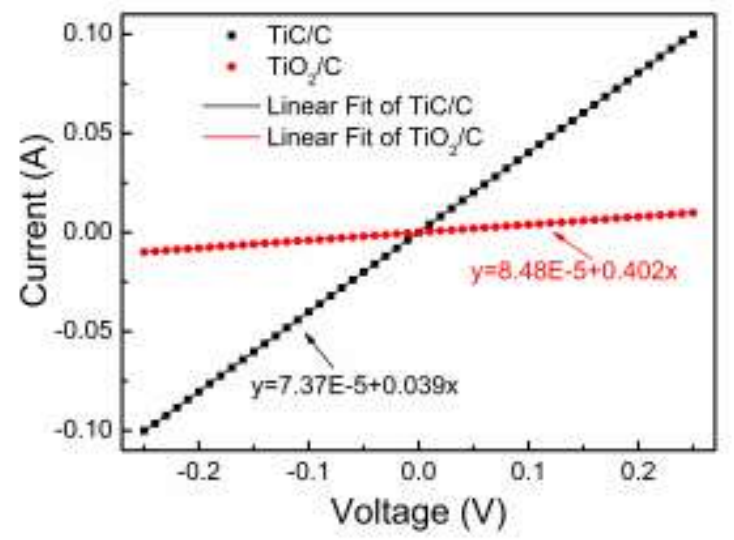

Figure S4. I-V characteristics determined from the two-point solid-state measurements of $\mathrm{TiO}_{2} / \mathrm{C}$ and $\mathrm{TiC} / \mathrm{C}$ QANFAs prepared on $\mathrm{Ti}$ and Ti6Al4V.The approximate resistances of the $\mathrm{TiO}_{2} / \mathrm{C}$ and TiC/C QANFAs are 25.6 $\Omega$ and 2.5 , respectively. 
Table S1. $\Delta E_{\mathrm{p}}$ values obtained from $\mathrm{TiC} / \mathrm{C}$ and $\mathrm{TiO}_{2} / \mathrm{C}$ QANFAs electrodes with a various resistance connected in series.

\begin{tabular}{ccccccccccccc}
\hline Electrode & \multicolumn{1}{c}{$\mathrm{TiC} / \mathrm{C}$} & \multicolumn{1}{c}{$\mathrm{TiO}_{2} / \mathrm{C}$} \\
\hline $\begin{array}{c}\text { Connected } \\
\text { resistance }(\Omega)\end{array}$ & 0 & 10 & 20 & 50 & 100 & 200 & 500 & 0 & 30 & 80 & 180 & 480 \\
$\Delta E_{p}(\mathrm{mV})$ & 59 & 62 & 70 & 82 & 105 & 145 & 245 & 70 & 85 & 110 & 151 & 255 \\
\hline
\end{tabular}

Table S2. Variation of $\Delta E_{p}$ and apparent $k_{0}$ in the $\mathrm{Ru}\left(\mathrm{NH}_{3}\right)_{6}{ }^{3+/ 2+}$ and dopamine redox

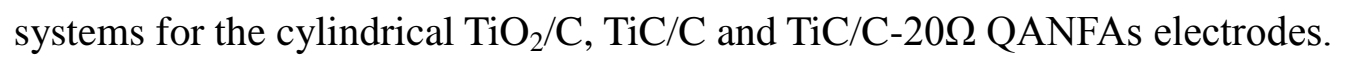

\begin{tabular}{ccccc}
\hline & Electrode & $\mathrm{Ru}\left(\mathrm{NH}_{3}\right)_{6}{ }^{3+/ 2+}$ & $\mathrm{Fe}^{3+/ 2+}$ & Dopamine \\
\hline \multirow{3}{*}{$\Delta E_{p}(\mathrm{mV})$} & $\mathrm{TiO}_{2} / \mathrm{C}$ & $67 \pm 2$ & $239 \pm 7$ & $32 \pm 1$ \\
& $\mathrm{TiC} / \mathrm{C}$ & $53 \pm 1.33$ & $235 \pm 5.67$ & $29 \pm 0.33$ \\
& $\mathrm{TiC} \mathrm{C}-20 \Omega$ & $69 \pm 2.33$ & $240 \pm 5.67$ & $31 \pm 1$ \\
$k_{0}\left(\mathrm{~cm} \mathrm{~s}^{-1}\right)$ & $\mathrm{TiO}_{2} / \mathrm{C}$ & 0.032 & $\sim 0.0006$ & 0.054 \\
& $\mathrm{TiC} / \mathrm{C}$ & $>0.18$ & $\sim 0.0007$ & $>0.18$ \\
& $\mathrm{TiC} / \mathrm{C}-20 \Omega$ & 0.025 & $\sim 0.0006$ & 0.13 \\
\hline
\end{tabular}

